UCRL-JC-127269

PREPRINT

\title{
Physical Characterization of Ultrashort Laser Pulse Drilling of Biological Tissue
}

\author{
M. D. Feit, A. M. Rubenchik, B.-M. Kim, L. B. Da Silva, \\ B. C. Stuart, M. D. Perry
}

This paper was prepared for submittal tothe

Fourth Conference on Laser Ablation

Pacific Grove, CA

July 20-25, 1997

July 21, 1997

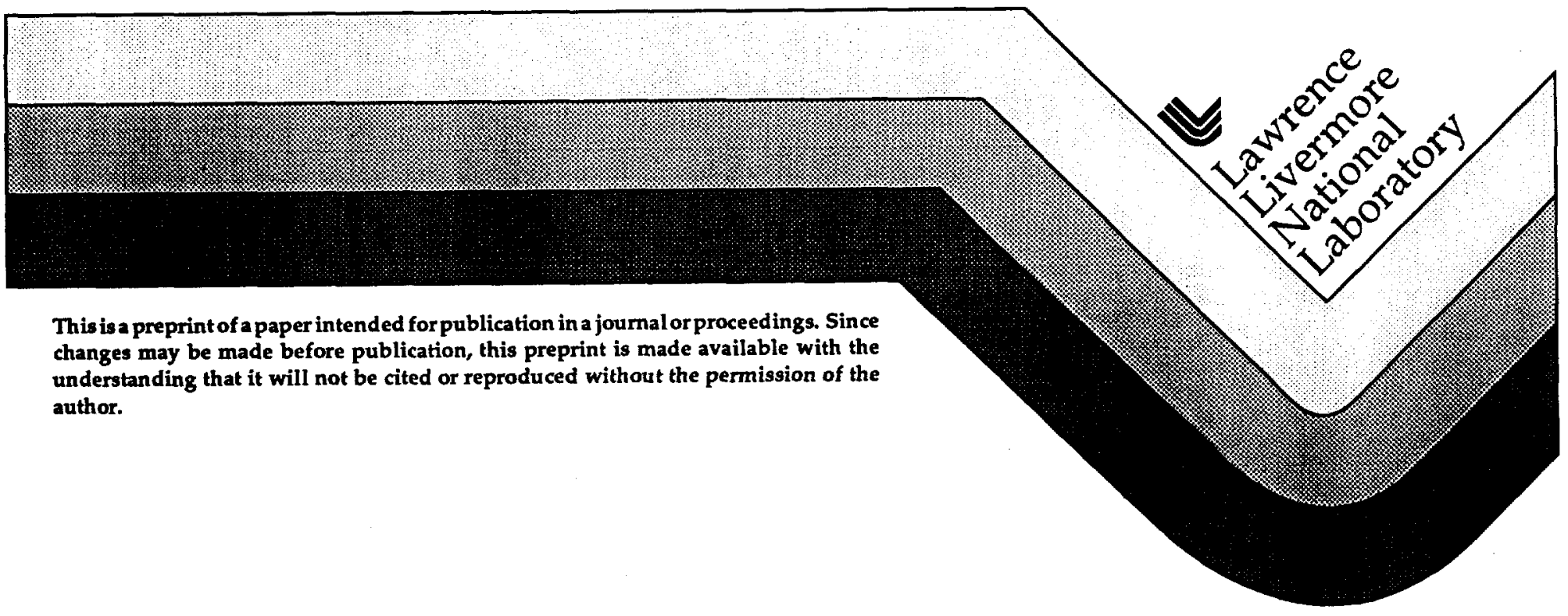




\section{DISCLAMER}

This document was prepared as an account of work sponsored by an agency of the United States Government. Neither the United States Government nor the University of California nor any of their employees, makes any warranty, express or implied, or assumes any legal liability or responsibility for the accuracy, completeness, or usefulness of any information, apparatus, product, or process disclosed, or represents that its use would not infringe privately owned rights. Reference herein to any specific commercial product, process, or service by trade name, trademark, manufacturer, or otherwise, does not necessarily constitute or imply its endorsement, recommendation, or favoring by the United States Government or the University of California. The views and opinions of authors expressed herein do not necessarily state or reflect those of the United States Government or the University of California, and shall not be used for advertising or product endorsement purposes. 


\title{
Physical Characterization of Ultrashort Laser Pulse Drilling of Biological Tissue*
}

M.D. Feit, A.M. Rubenchik, B.-M. Kim, L.B. Da Silva, B.C. Stuart, and M.D. Perry

\author{
Lawrence Livermore National Laboratory
}

PO Box 808, mail stop L-399

Livermore, CA 94550

feit1@llnl.gov, rubenchik1@llnl.gov, kim12@llnl.gov, dasilva1@llnl.gov, perry10@llnl.gov

\begin{abstract}
Ultrashort laser pulse ablation removes material with low energy fluence required and minimal collateral damage. The ultimate usefulness of this technology for biomedical application depends, in part, on characterization of the physical conditions attained and determination of the zone of shockwave and heat affected material in particular tissues. Detailed numerical modeling of the relevant physics (deposition, plasma formation, shockwave generation and propagation, thermal conduction) are providing this information. A wide range of time scales is involved, ranging from picosecond for energy deposition and peak pressure and temperature, to nanosecond for development of shockwave, to microsecond for macroscopic thermophysical response.
\end{abstract}

* Work performed at Lawrence Livermore National Laboratory under the auspices of the U.S. Department of Energy under contract No. W-7405-ENG-48. 


\section{Introduction}

There is a need for precise tissue removal with minimal collateral thermal/mechanical damage in many laser-surgical applications including neurosurgery, dentistry and cardiology. Work done at LLNL[1] suggests that an ultrashort (less than several ps) pulse laser ablation system can meet this need. We are engaged on an experimental/theoretical project to develop the necessary scientific knowlede of the mechanisms involved and then to apply this knowledge. We are simultaneously extending our understanding of the laser ablation process while developing ancillary systems necessary for practical applications [2]. The ablation rate is approximately $1 \mu \mathrm{m} / \mathrm{pulse}$ so a high repetition rate is necessary to reach practical material removal rates. Because of the high repetition rate, a sophisticated feedback system possibly including tissue fluorescence, plasma emission luminescence or optical coherence tomography (OCT) is desirable for precise control.

The theoretical component of this project is focused on developing models for fundamental laser-tissue interaction, incorporating such descriptions in numerical models[3] and applying these models to experimental design and data analysis. Theoretical studies also are needed to evaluate delivery systems such as hollow core optical fibers. Experiments will measure ablation rates, crater formation and other physical quantities such as temperature and pressure in actual laser-tissue interactions. An iterative cycle of theoretical design and experiment will be used to develop the final system design and improve the accuracy of theoretical models. The present paper describes studies of laser energy deposition in nominally transparent media (eg water), thermal and shockwave generation, the range and degree of thermal loading and the thermal effect of high repetition rates, and crater formation. The relative advantages of ps duration pulses compared to ns duration pulses will be demonstrated.

\section{Laser Energy Deposition}


The basic physical description of ultrashort laser pulse energy deposition in insulators was developed during our studies of the onset of laser induced damage in fused silica and other materials due to interaction with intense ultrashort laser pulses [4]. This picture was extended to tissue in [5]. Three noteworthy features should be pointed out.

First, there is insufficient time during the laser pulse for the usual optical breakdown, based on electrons contributed by trace impurities or chromophores, to occur. Instead, higher intensities are reached at which nonlinear multiphoton absorption and ionization takes place. This process is much more deterministic and localized than linear absorption since it depends on a high power of the laser intensity. Fig. 1 shows the case of a $100 \mathrm{fs}$ pulse where nonlinear absorption provides a high enough density of conduction electrons for an ensuing avalanche (collisional ionization) which reaches the critical plasma density.

Secondly, the laser energy is deposited in electrons much faster than the electronphonon equlibration time or any hydrodynamic time. Most of this energy ultimately appears as kinetic energy of ejected material rather than as bulk thermal energy. As a result, much less collateral damage (heat affected zone) occurs.

The third effect is related to the second. For longer (say ns) pulses, plasma created by the early portion of the pulse expands and strongly absorbs incoming laser light. The plasma effectively shields the underlying material. In the ultrashort case, in contrast, the plasma doesn't have time to expand and instead, at high intensities, acts as a mirror reflecting a portion of the incoming light [6].

\section{Initial Pressure and Temperature}

When viewed on the scale of laser pulse energy deposition (nanometers in depth, ps in time), detailed calculations indicate a peak pressure near $1 \mathrm{Mbar}$ (Fig. 2a) and temperatures more than $20 \mathrm{eV}$ (Fig. 2b). These calculations are for a $0.5 \mathrm{ps}, 1.5 \mathrm{~J} / \mathrm{cm}^{2} 1.06$ $\mu \mathrm{m}$ pulse in water. Although the initial temperature and pressure are high, a high fraction of the absorbed energy is removed by the ejecta. The subsequent shocking of the bulk 
material is much less than that resulting from a ns pulse which removes a comparable amount of material. This is illustrated in Fig.(3) where the scale of Fig. 2a is expanded by a factor of 1000 in both space and time, ie $\mu \mathrm{m}$ and ns. Fig. (3a) shows the shock wave generated by the ultrashort pulse. Despite the high initial pressure, the peak pressure has dropped below $5 \mathrm{Kbar}$ after penetrating only about $10 \mu \mathrm{m}$. In contrast, the shock

generated by a $5 \mathrm{~ns} 25 \mathrm{~J} / \mathrm{cm}^{2}$ pulse is shown in Fig.(3b). Not only does the shock penetrate much further into the material, it also, of course, lasts much longer in time. This indicates the advantage of ultrashort pulses for minimizing collateral damage.

\section{Effect of High Repetition Rate Ablation}

Consider laser ablation at repetition rate $\mathrm{p}$ and pulse duration $\tau$. We assume the rate

is small enough so that $p \tau<<1$. Away from the surface where the laser energy is deposited in a thin zone, and for times long compared to the pulse length, it is reasonable to find the temperature rise from the heat equation. We neglect material removal in the following, ie measure depth $\mathrm{z}$ from the actual surface. Energy is deposited in a periodic sequence of delta function pulses at the surface. Because energy is deposited in a "pancake" shape, heat diffusion is initially one dimensional, into the material. For times long enough so that the thermal diffusion length is comparable to or larger than the deposition spot size, heat conduction becomes three dimensional and the temperature drops rapidly. The axial temperature after many pulses is given by

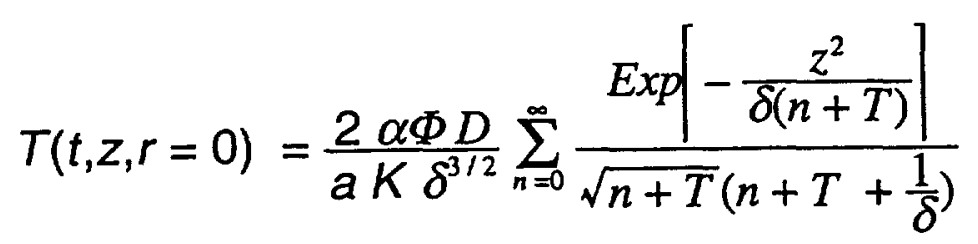

Here a is the radius of the heat deposition spot (assumed Gaussian), $\Phi$ is the incident fluence a fraction $\alpha$ of which is absorbed as thermal energy, $\mathrm{D}$ is the thermal diffusivity $\mathrm{K}$ is the thermal 
conductivity, and $\delta=4 \mathrm{D} /\left(\mathrm{pa}^{2}\right)$ is the square of the ratio of diffusion length during a pulse repetition time to the beam radius. Here $T$ is the time since the last pulse measured in units of $1 / p$, ie $T=\tau_{p}<1$. The term $n=1$ gives effect of the previous pulse, $n=2$ the pulse before that and so on.

It should be noted that the term multiplying the summation scales as $\Phi \mathrm{a}^{2} \mathrm{p}^{3 / 2}$, ie as the pulse energy times the $3 / 2$ power of the rep rate.

Near the surface, the temperature varies periodically. Further in, ie. beyond a thermal diffusion length corresponding to the period, the material responds to the temporal average input heat flux. Poor conductors reach higher temperatures than do good conductors for the same thermal loading. In this case, evaporative cooling may play an important role.

If the material has a finite thickness $h$, and it is assumed there is no heat flow out of the rear surface, then the additional boundary condition at the rear surface of the vanishing of the temperature gradient leads to a somewhat more complicated solution. In this case, the exponential decay in $z^{2}$ is replaced by an infinite sum over over image sources. That is,

$$
T(t, z, r=0)=\frac{2 \alpha \Phi D}{a K \delta^{3 / 2}} \sum_{n=0}^{\infty} \frac{\left.\sum_{s=0}^{\infty} \operatorname{Exp}\left\lceil-\frac{(z+2 s h)^{2}}{\delta(n+T)}\right\rceil+\operatorname{Exp} \mid-\frac{(z-2(s+1) h)^{2}}{\delta(n+T)}\right\rceil}{\sqrt{n+T}\left(n+T+\frac{1}{\delta}\right)}
$$

When $h$ is very large, only a single term of the new sum contributes and the previous solution for an infinitely thick medium is obtained.

Our calculations indicate that repetition rates of over $1 \mathrm{Khz}$ should not result in serious thermal problems in most cases.

\section{Crater Formation and Propagation Effects}

Since each laser pulse removes only a small amount of material, crater formation involves a series of ablation events. We previously [1] showed that one can account for the shape of the crater based on the concept that the crater walls steepen until the effective 
normal intensity is reduced to the threshold value. This means that a late pulse has to propagate through a channel in the ablated material. For a deep hole, this is equivalent to propagation in a waveguide with (perhaps) slowly narrowing walls. An interesting example of a propagation effect is the polarization dependence of laser energy coupling to the walls.

The coupling of laser light into the tissue can depend on the direction of the electric field. This is due to the difference in boundary conditions at a surface between an electric field parallel to or perpendicular to the surface. In our case, the surface is the laser drilled hole or cut. The exact values for energy coupling depend on the dielectric constants involved, but the trend can be seen in Fig.(4) which shows that energy absorption from a laser beam propagating in an infinitely long planar cut is largest for $\mathrm{S}$ polarization, ie for electric field pointing across the cut. These results imply a connection between incident intensity and the depth of a hole that could be drilled in an infinite medium. Also note that the coupling becomes stronger for a narrower cut (assuming the cut is empty).

\section{Conclusions}

We have good theoretical understanding of ultrashort laser pulse energy deposition in dielectrics, plasma formation, plasma absorption and reflection on the ps time scale. We can reliably model the generation and propagation of shockwaves and thermal conduction on the ns time scale. The separation of the energy deposition timescale from thermal conduction and hydrodynamic timescales results in advantages for ultrashort pulse laser ablation such as small required energy and minimal collateral damage to surrounding tissue. Future work will include bulk material response.

references

[1]This work was first published in a series of conference proceedings including "Comparison of soft and hard tissue ablation using ultrashort laser pulses", L.B. daSilva, B.C. Stuart, P. Celliers, M.D. Feit, N.J. Heredia, S.M. Herman, D.L. Mathews, M.D. 
Perry and A.M. Rubenchik, Biomedical Optics '96, SPIE 2681-26, (San Jose, CA 1996); "Computational modeling of ultrashort pulse ablation of enamel", R.A. London, D.S. Bailey, D.A. Young, M.D. Feit, A.M. Rubenchik, J. Neev, Biomedical Optics '96, SPIE 2681-31, (San Jose, CA 1996); "Unique Aspects of Ultrashort Laser Pulse Energy Deposition in Tissue", M.D. Feit, A.M. Rubenchik, B.W. Shore, Biomedical Optics '96, SPIE 2672-36, ( San Jose, CA 1996); “ Dental tissue processing with ultra-short pulse laser", A.M. Rubenchik, L.B. daSilva, M.D. Feit, S.Lane,R.London, M.D. Perry, B.C. Stuart, J. Neev, Biomedical Optics '96, SPIE 2672-34, (San Jose, CA 1996);

"Hydrodynamic model for ultrashort-pulse albation of hard dental tissue," R. A. London, D. S. Bailey, D. A. Young, W.E. Alley, M. D. Feit et al., , Biomedical Optics '96, SPIE 2672, 231-242 (1996) .

[2] "Optical Fluorescence Feedback Signal for Ultrashort Laser Pulse Tissue Ablation", B.-M. Kim, L.B. Da Silva, M.D. Feit, A.M. Rubenchik

[3] "Numerical Simulation of ultra-short laser pulse energy deposition and transport for material processing", _A. M. Rubenchik, M.D. Feit, M. D. Perry, and J. T. Larsen ( this conference)

[4] "Laser induced damage in dielectrics with nanosecond to subpicosecond pulses", B.C. Stuart, M.D. Feit, A.M. Rubenchik, B.W. Shore and M.D. Perry, Phys. Rev. Lett 74, 2248-51 (M 1995); "Nanosecond to femtosecond laser-induced breakdown in dielectrics", B.C. Stuart, M.D. Feit, A.M. Rubenchik, B.W. Shore and M.D. Perry, Phys Rev B 53, 1749-61 (1996); "Optical ablation by high-power short-pulse lasers" , B.C. Stuart, M.D. Perry, R.D. Boyd, J.A. Britten, B.W. Shore, M.D. Feit and A.M. Rubenchik, JOSA B 13, $459-468$ (1996)

[5] "Ultrashort Pulse Lasers for Hard Tissue Ablation", J. Neev, L.B. DaSilva, M.D. Feit, M.D. Perry, A.M. Rubenchik and B.C. Stuart, IEEE Selected Topics in Quantum Electronics 2, 790-800 ( 1996); "Plasma mediated ablation of biological tissues with nanosecond to femtosecond laser pulses: relative role of linear and nonlinear absorption", 


\author{
A.A. Oraevsky L.B. DaSilva, A.M. Rubenchik, M.D. Feit, M.E. Glinsky, M.D. Perry, \\ B.M. Mammini, W. Small IV and B.C. Stuart, IEEE Selected Topics in Quantum \\ Electronics 2, 801-9 ( 1996) \\ [6] "Plasma Mirrors", M.D. Perry, V. Yanovsky, M.D. Feit, A.M. Rubenchik, LLNL \\ report UCRL-JC-125376, 1997
}

\title{
Figure Captions
}

Fig. 1 : Electron density (left scale) resulting from $100 \mathrm{fs}$ pulse whose intensity is indicated by dashed curve and right scale. A high density is produced by multiphoton ionization during the first half of the pulse. Subsequent collisional ionization then yields further ionization.

Fig. $2 \mathrm{a}$ - On the spatial scale (nm) and temporal scale (ps) of laser energy deposition, we calculate peak pressures near one Mbar and temperatures of $20 \mathrm{eV}$ for a $0.5 \mathrm{ps} 1.5 \mathrm{~J} / \mathrm{cm}^{2}$ pulse in water.

Fig. 3a On spatial and temporal scales 1000 times larger than that of Fig. 2, the shockwave corresponding to ultrashort pulse is seen to decay after penetrating a few mm. The shock generated by a 5 ns pulse, however, lasts much longer and penetrates much further.

Fig. 4 The absorption of laser energy at a cut, modeled as a planar defect, depends on the polarization of the light (S corresponds to electric field across the cut). 

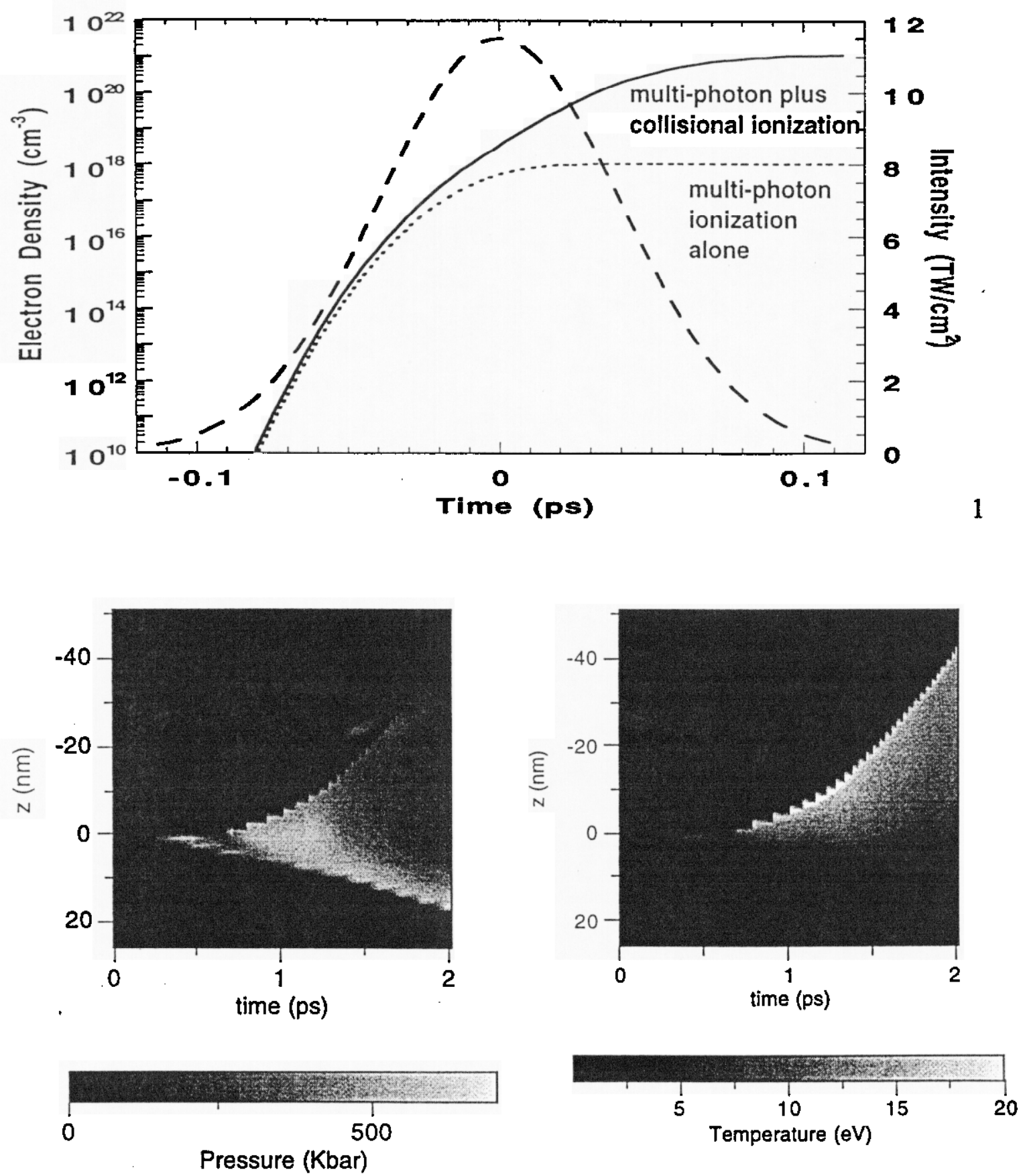

Fig. 2a

Fig. 2b 

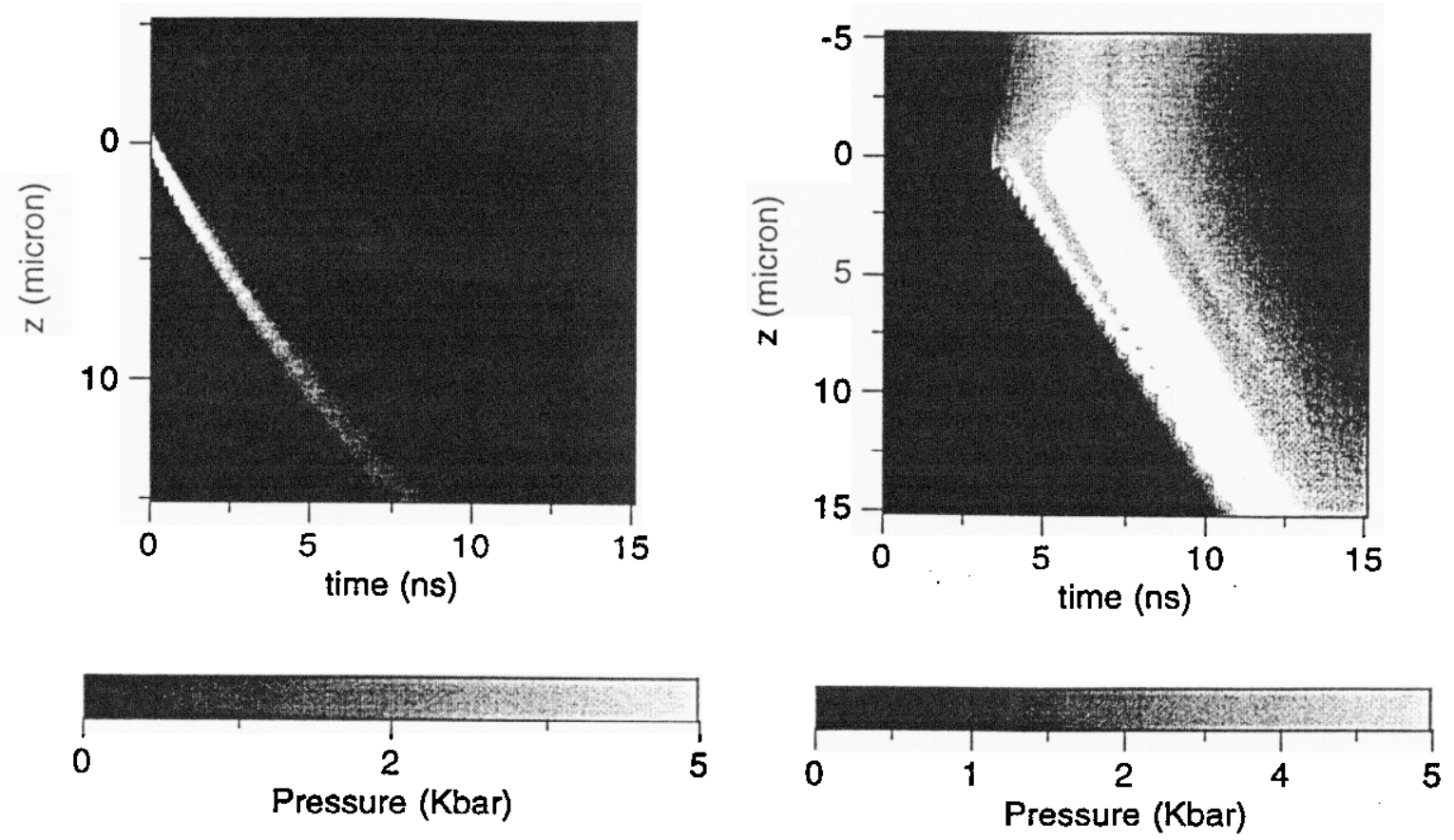

Fig. 3a

Fig. 3b 


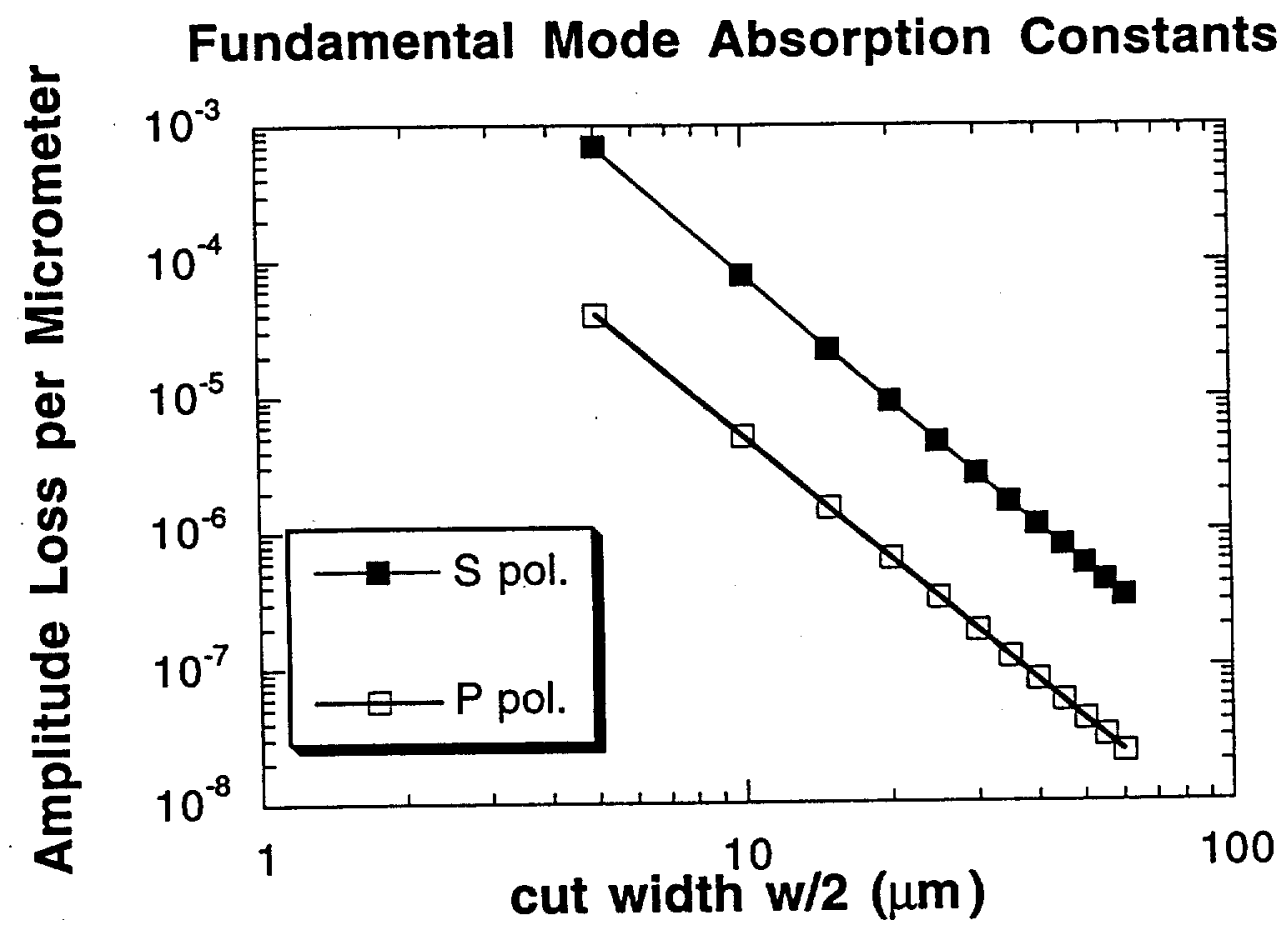

Fig. 4 


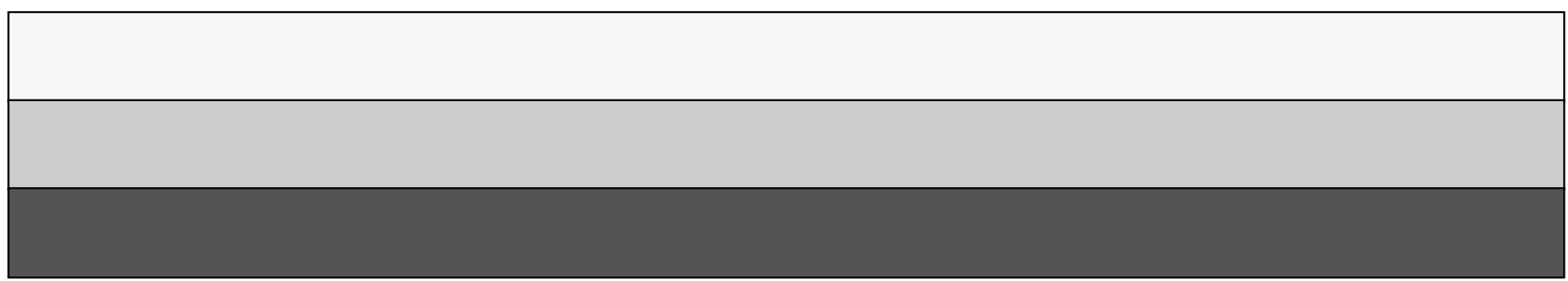

